\title{
Characterization of Mycobacterium tuberculosis Beijing isolates from the Mediterranean area
}

\author{
M Alonso 1,2,3, N Alonso Rodriguez1 , C Garzelli4 ${ }^{4}$ M Martínez Lirola5, M Herranz1,3, S Samper 3,6, MJ Ruiz Serrano 1,3, \\ E Bouza 1,3 and D García de Viedma*1,2,3
}

\begin{abstract}
Background: The Beijing lineage of Mycobacterium tuberculosis is causing concern due to its global distribution and its involvement in severe outbreaks. Studies focused on this lineage are mainly restricted to geographical settings where its prevalence is high, whereas those in other areas are scarce. In this study, we analyze Beijing isolates in the Mediterranean area, where this lineage is not prevalent and is mainly associated with immigrant cases.

Results: Only 1\% ( $\mathrm{N}=26)$ of the isolates from two population-based studies in Spain corresponded to Beijing strains, most of which were pan-susceptible and from Peruvian and Ecuadorian patients. Restriction fragment length polymorphism typing with the insertion sequence IS6110 identified three small clusters (2-3 cases). Mycobacterial interspersed repetitive unit-variable number tandem repeat typing (MIRU-15) offered low discriminatory power, requiring the introduction of five additional loci. A selection of the Beijing isolates identified in the Spanish sample, together with a sample of Beijing strains from Italy, to broaden the analysis context in the Mediterranean area, were assayed in an infection model with THP-1 cells. A wide range of intracellular growth rates was observed with only two isolates showing an increased intracellular replication, in both cases associated with contained production of TNF-a. No correlation was observed between virulence and the Beijing phylogenetic group, clustered/orphan status, or resistance. The Beijing strain responsible for extensive spread on Gran Canaria Island was also identified in Madrid, but did not lead to secondary cases and did not show high infectivity in the infection model.

Conclusions: The Beijing lineage in our area is a non-homogeneous family, with only certain highly virulent representatives. The specific characterization of Beijing isolates in different settings could help us to accurately identify the virulent representatives before making general assumptions about this lineage.
\end{abstract}

\section{Background}

Tuberculosis (TB) is one of the main infectious causes of death worldwide, with more than 9 million new cases of active disease every year and nearly 2 million deaths [1]. Mycobacterium tuberculosis (MTB) is the causative agent of most TB cases, and its ability to spread and the outcome of infection depend on epidemiological, host, and bacterial factors [2].

The MTB genome is highly conserved, but several large sequence polymorphisms defining different genetically related lineages have been identified. Among them, the Beijing family can be identified rapidly and reliably by several genetic features. These include a characteristic

* Correspondence: dgviedma2@gmail.com

1 Servicio de Microbiología Clínica y Enfermedades Infecciosas, Hospital Gregorio Marañón, Madrid, Spain

Full list of author information is available at the end of the article spoligotype with exclusive deletion of spacers 1-34 (the so-called RD207 deletion) [3], an intact open reading frame in the $p k s 15 / 1$ gene [4], and deletion of the genomic region RD105, which define the Beijing family as a separate lineage within MTB [5].

The Beijing lineage is causing major concern worldwide $[6,7]$ because its worldwide spread and involvement in several TB outbreaks, some of them involving drug-resistant strains [8]. The Beijing lineage is generally considered to be associated with drug-resistance, although this association has not been found in all geographic settings $[7,8]$. The proportion of Beijing strains differs, being low in Western Europe, although a slight increase in the number of Beijing strains has been detected over time [6]. The presence of this lineage in the population has been associated with the recent increase in the number of TB cases 
among immigrants observed in several areas, including the Mediterranean $[8,9]$.

The wide distribution of Beijing strains suggests that members of this phylogenetic lineage are better adapted to infect and cause disease in humans than other MTB families, and there are reports indicating that Beijing strains show higher replication rates and more virulent phenotypes than other MTB lineages in both in vitro and in vivo models $[10,11]$. The infective success of this lineage seems to be associated with its effect on the immune response, in that it can control the release of the macrophage-derived cytokines that play a central role in directing the immune response towards a non-protective Th2 phenotype $[12,13]$.

The incidence of the Beijing lineage in Spain is low, although in recent years it has been increasing due to immigration [9]. The profile of nationalities of the immigrants infected by Beijing isolates differs from that observed in other countries, and South American cases are the most common. The impact of the importation of Beijing isolates to Spain was described in the 1990s on Gran Canaria Island, where an extensive outbreak involving this lineage was detected after a Beijing isolate was identified in an immigrant [14].

Studies analyzing the Beijing lineage are scarce in the Mediterranean area $[15,16]$. We explored whether specific genotypic and phenotypic features could be found for the Beijing strains isolated in a context where this clade is not endemic, but imported by immigrants whose origin (mainly Peru and Ecuador) is different from that found in other settings.

\section{Results}

\section{Identification and characterization of Beijing isolates}

Of the 2391 isolates analyzed in the Spanish sample, 26 $(1.09 \%)$ were identified as members of the Beijing lineage according to the criteria reported in the Methods section. In particular, nineteen showed deletion of the spacers 134 and the characteristic hybridization pattern of spacers $35-43$, and the remaining seven corresponded to variant "Beijing-like" spoligotypes.

In order to verify the spoligotyping-based identification of Beijing strains and to refine the genetic characterization, the $p k s 15 / 1$ gene and the RD105, RD181, RD150, and RD142 were analyzed. The $p k s 15 / 1$ gene, which is generally considered a marker for $M$. tuberculosis strains of Asian origin [4,17], was sequenced in all 26 isolates in order to rule out deletions, and in all cases this gene was intact (Table 1). The genomic deletion RD105, which phylogenetically defines the Beijing family [5], was found in all 26 (Table 1). On the basis of the polymorphisms associated with genomic deletions RD181, RD150, and RD142, previously defined for the Beijing lineage by Reed et al[18], all of the isolates belonged to phylogenetic group 3 except one, which belonged to group 4 .

By origin, 22 of the 26 isolates were from foreign-born cases $(84.6 \%)$ of nine different nationalities, the most frequent being Peruvians and Ecuadorians (42\%). The remaining four Beijing isolates corresponded to autochthonous cases (Table 1).

The drug susceptibility tests showed that 23 of the 26 isolates were pan-susceptible, two were isoniazid-resistant, and one was multidrug-resistant (Table 1).

\section{Genotyping analysis}

The IS6110-RFLP analysis revealed 21 different genotypes (9-22 IS6110 copies). Seven isolates (26.9\%) were grouped in two clusters of three and four cases each. Nineteen isolates $(73.1 \%)$ were unclustered and considered orphan cases (Figure 1A). The isolates involved in cluster 2 (C2) shared an identical IS6110-RFLP pattern with those involved in the Gran Canaria outbreak [14].

The MIRU-15 analysis identified 18 different genotypes among the Beijing isolates. Thirteen isolates (50\%) were grouped in five clusters of two or three cases. The remaining isolates corresponded to orphan cases (Figure 1B). If we compare RFLP and MIRU-15 data, it is noteworthy that two representatives of cluster $1(\mathrm{C} 1)$, defined by RFLP, were split by MIRU-15, and three of the clusters defined by MIRU-15 grouped isolates that had been considered orphan by RFLP. Only the $\mathrm{C} 2$ cluster defined by RFLP remained intact after MIRU analysis. Regarding the isolates clustered in C2, which shared the RFLP pattern with the isolate involved in the Gran Canaria outbreak, we also pursued to compare the MIRU-15 data. With this aim, a selection of Gran Canaria outbreak isolates, sharing also the susceptibility pattern with those form Madrid, were analyzed and an identical MIRU-15 type was shared by the representatives from Madrid and Gran Canaria.

After observing the low discrimination of MIRU-15, five new VNTR loci (QUB11a, QUB3232, QUB18, VNTR3820, and VNTR4120) were added; they were all selected due to their high discriminatory values in different studies focused on Beijing isolates [19,20]. The discriminatory power of this MIRU-15+5 set was markedly higher than that of MIRU-15, and was even higher than that of RFLP. Twenty-five different genotypes were identified from the 26 isolates analyzed. Four of the five MIRU-15 clusters were sub-divided by MIRU-15+5 (Figure $1 \mathrm{C}$ ), and only the $\mathrm{C} 1$ cluster defined by MIRU-15 remained intact.

\section{Infectivity characterization i) Intracellular growth in THP-1 cells}

Eight of the 26 Beijing isolates characterized in the Spanish sample (1-8) were selected to assure a suitable vari- 
Table 1: Epidemiological and genetic features of Beijing strains

\begin{tabular}{|c|c|c|c|c|}
\hline Total & Nationality & Drug susceptibilitya & pks15/1 geneb & RD105c \\
\hline 8 & Ecuador & $\mathrm{S}$ & + & - \\
\hline 3 & Peru & $1 \mathrm{INH}-\mathrm{R} / 2 \mathrm{~S}$ & + & - \\
\hline 2 & Morocco & $1 \mathrm{INH}-\mathrm{R} / 1 \mathrm{~S}$ & + & - \\
\hline 4 & China & $\mathrm{S}$ & + & - \\
\hline 1 & Armenia & $\mathrm{S}$ & + & - \\
\hline 1 & Moldavia & $\mathrm{S}$ & + & - \\
\hline 1 & Ukraine & MDR & + & - \\
\hline 1 & South Africa & $\mathrm{S}$ & + & - \\
\hline 1 & Russia & $\mathrm{S}$ & + & - \\
\hline 4 & Spain & $S$ & + & - \\
\hline
\end{tabular}

ability according to different features: nationality of the cases (6 nationalities), drug susceptibility (2 resistant and 6 susceptible), number of IS6110 copies (9-22) and phylogenetic group (groups 3 and 4) (Table 2). The strain responsible for the outbreak on Gran Canaria Island was also included (isolate no. 1). To widen the geographic setting to the Mediterranean area and to increase both the number of Beijing representatives analyzed and the number of isolates involved in clusters, we included to the Spanish Beijing representatives, eight additional Beijing isolates (9-16) from Tuscany, Italy (Table 2). As controls, we included the virulent reference strain $\mathrm{H} 37 \mathrm{Rv}$ and a non-Beijing representative orphan strain.

A wide range of intracellular growth rates was detected among the Beijing isolates assayed (Figure 2). Two isolates showed the highest intracellular growth rates, which differed significantly $(\mathrm{P}<0.05)$ from the others. There were no significant differences in growth rate among the remaining isolates, including control strain $\mathrm{H} 37 \mathrm{Rv}$ and the non-Beijing orphan strain. No correlation was found between the epidemiological status of the isolates (clustered/unclustered) and the intracellular growth rates. The isolate responsible for the outbreak on Gran Canaria Island was included, although it did not show increased intracellular replication.

\section{ii) Cytokine production}

We studied the immunoregulatory profile of cytokines secreted in THP-1 cells infected with six isolates selected as representatives of the different intracellular growth rates in the previous assay, including $\mathrm{H} 37 \mathrm{Rv}$. In all cases, the infection by MTB increased the TNF- $\alpha$ production compared to the values observed in the non-infected control. The TNF- $\alpha$ production dynamics along the infection differed among the isolates analyzed. Four isolates induced a peak level of TNF- $\alpha$ at day 1 after infection, and this was followed by a rapid decrease in secretion (Figure 3A). The other two isolates were those that had the highest growth rates in the THP-1 assay. In these isolates, TNF- $\alpha$ production followed a different pattern, namely, production of TNF- $\alpha$ was contained from the start of infection, and was significantly lower than that induced by the remaining isolates at day 1 after infection. TNF- $\alpha$ levels in these isolates continued to decrease throughout the infection (Figure 3A).

The IL-10 secretion profiles in THP-1 cells infected with all the Beijing isolates were similar to the noninfected controls at early stages of infection; IL-10 production was not detected up to day 1 after infection (Figure $3 \mathrm{~B}$ ). However, from this point, the IL-10 production dynamics differed among the isolates analyzed with peak levels occurring at day 4. IL-10 levels subsequently decreased in all of the isolates except two, in which production increased until infection resolved (Figure 3B). These two isolates corresponded to those which contained production of TNF- $\alpha$ and showed the highest growth rates in THP-1-infected cells. Thus, a correlation was found between intracellular replication, production of TNF- $\alpha$, and the immunoregulatory response through IL-10 in THP-1 infected cells.

Both of the strains with a differential behaviour in the THP-1 model corresponded to South American orphan cases: one belonged to phylogenetic group 4 and was isoniazid-resistant, and the other belonged to phylogenetic group 3 and was pan-susceptible and displayed the lowest number of IS6110 copies (nine).

The Beijing isolate responsible for the TB outbreak on Gran Canaria Island was not distinguishable from other isolates. It had an average intracellular growth rate and 


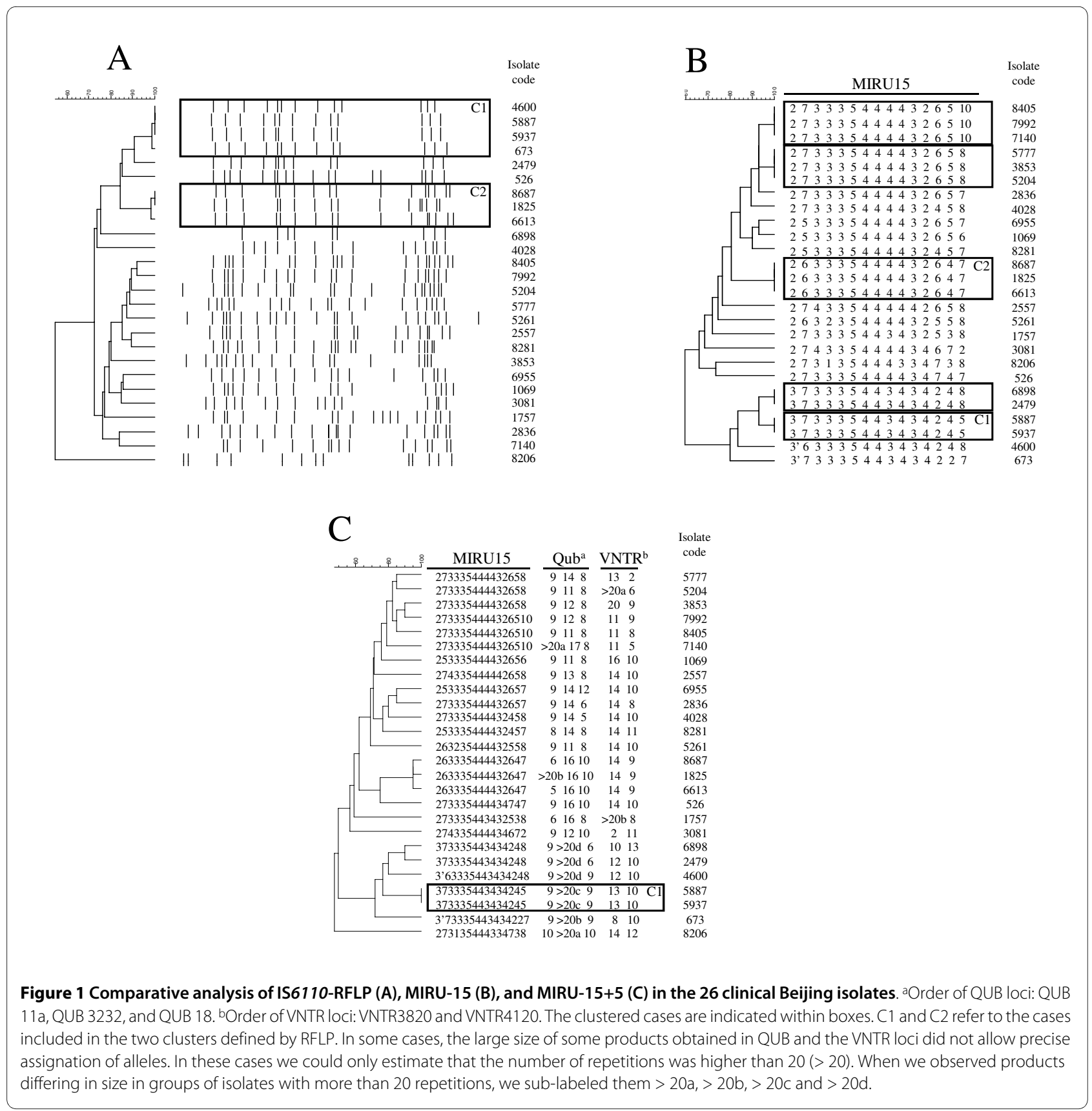

did not control TNF- $\alpha$ levels at early stages of the infection.

When we considered the cluster/orphan status of the isolates, analysis of intracelullar growth rates and cytokine expression profiles did not reveal a correlation between cluster/orphan status and infective behaviour in the THP-1 model.

\section{Discussion}

The worldwide distribution of the Beijing lineage has been well documented [6-8], being this genotype highly prevalent (70-80\% in total isolates) in East Asia (China,
Korea, Japan, etc). However, the proportion of Beijing strains in Western Europe is low. In two countries of the Mediterranean area, Italy and Spain, the marked increase in the number of immigrants in recent years has led to an increase in the numbers of TB cases that can be attributed to imported strains. In Madrid (Spain) and Tuscany (Italy) during the period 2004-2006, slightly more than $40 \%$ of all cases of TB were detected in immigrants $[15,21]$. We characterized the genotypic and phenotypic features of the Beijing lineage in a setting where it is not frequently isolated and where it is mostly detected in immigrant cases. 
Table 2: Beijing strains assayed in THP-1 cells

\begin{tabular}{|c|c|c|c|c|c|c|c|}
\hline Isolate code & $\begin{array}{c}\text { Year of } \\
\text { isolation }\end{array}$ & Strain No. & Nationality & Drug susceptibilitya & IS6110 copy no.b & $\begin{array}{c}\text { Clustered/ } \\
\text { Orphan }(+/-)^{c}\end{array}$ & RD Groupd \\
\hline 8687 & 2002 & 1 & Spain & $S$ & 16 & + & 3 \\
\hline 5204 & 2005 & 2 & China & $S$ & 22 & - & 3 \\
\hline 7992 & 2005 & 3 & Ecuador & S & 20 & - & 3 \\
\hline 8281 & 2004 & 4 & Armenia & $S$ & 21 & - & 3 \\
\hline 6955 & 2003 & 5 & Moldavia & $S$ & 16 & - & 3 \\
\hline 6898 & 2005 & 6 & Ecuador & $S$ & 9 & - & 3 \\
\hline 5261 & 2006 & 7 & Peru & INH-R & 22 & - & 4 \\
\hline 673 & 2006 & 8 & Ecuador & $S$ & 13 & + & 3 \\
\hline 1819 & 2005 & $9^{*}$ & Brazil & S & NA & NA & 3 \\
\hline 1884 & 2005 & $10^{*}$ & Peru & $S$ & NA & NA & 3 \\
\hline 1284 & 2004 & $11^{*}$ & Italy & INH-R SM-R & 17 & + & 3 \\
\hline 1538 & 2004 & $12^{*}$ & Peru & $S$ & 20 & + & 3 \\
\hline 1409 & 2004 & $13^{*}$ & China & $S$ & 18 & + & 3 \\
\hline 1254 & 2003 & $14^{*}$ & China & $S$ & 21 & + & 3 \\
\hline 838 & 2002 & $15^{*}$ & China & $S$ & 11 & - & 2 \\
\hline 1149 & 2003 & $16^{*}$ & Chile & $S$ & 9 & + & 2 \\
\hline
\end{tabular}

a INH-R, isoniazid-resistant; SM-R, streptomycin-resistant; S, pan-susceptible.

b Number of bands identified by RFLP. NA, not available.

c + and - indicate clustered/orphan status of the strain. NA, not available.

d Phylogenetic classification according to the presence or absence of the RD181, RD150, and RD142 genomic regions, according to Reed et al [18].

* Isolates from Tuscany.

Spoligotyping, sequencing of $p k s 15 / 1$, and analysis of the presence of the RD105 region revealed a low representativeness of this lineage in our population, as previously described in Central and Western Europe [8,9,22]. These studies also showed that Beijing strains in our area are mainly found in immigrants (ie, around $85 \%$ of our isolates were from immigrants, mostly Peruvians and Ecuadorians). This is consistent with the results of studies which report that the Beijing lineage was also imported to Europe via South America [23,24].

The Beijing lineage is generally considered to be associated with drug-resistant phenotypes, although this may not be true for all geographic settings $[7,8]$ and most of the Beijing strains in our study were susceptible. In fact, drug-resistant but also pan-susceptible strains have been associated with TB outbreaks [25] and it has recently been proposed that mainly atypical variants of Beijing strains are those linked to resistance [26].

IS6110-RFLP based genotyping was performed in order to establish a molecular epidemiological profile for the Beijing strains in the Spanish sample. Nineteen representative patterns of the Beijing genotype have been reported, and most of them have a high IS6110 copy number (15-26) [6,27]. The wider range of IS6110 copy numbers-- 9 to 22--alerts to the existence of Beijing iso- lates without a high number of IS6110 copies. The RFLP patterns of a 5 -year population based sample enabled us to define two clusters including 7 of the 26 Beijing isolates of the study (26.9\%); the first cluster contained three Ecuadorian immigrants and an autochthonous case, and the second contained three autochthonous patients. The members of the latter group shared an identical IS6110RFLP pattern with the one involved in the TB outbreak on Gran Canaria Island in the 1990s [14]. In our study, MIRU-15 was less discriminatory (13 of 26 isolates in five clusters) than RFLP. Recently, new hypervariable loci have been evaluated to increase the discriminatory capacity of the 15-loci VNTR typing method in the Beijing lineage $[19,20,28,29]$. A selection of them together with the 15-loci set, increased the discriminatory power to values even higher than those of RFLP.

The distribution of the Beijing lineage in different geographic areas and its ability to disseminate suggest that this phylogenetic lineage is better adapted to infect and cause TB in humans than other genetic lineages of MTB. It has been associated with high virulence and rapid growth in both in vitro and in vivo infection models $[10,11,30]$. These features are considered to be behind the success of Beijing strains, which is a consequence of their control over the immune response [12]. 


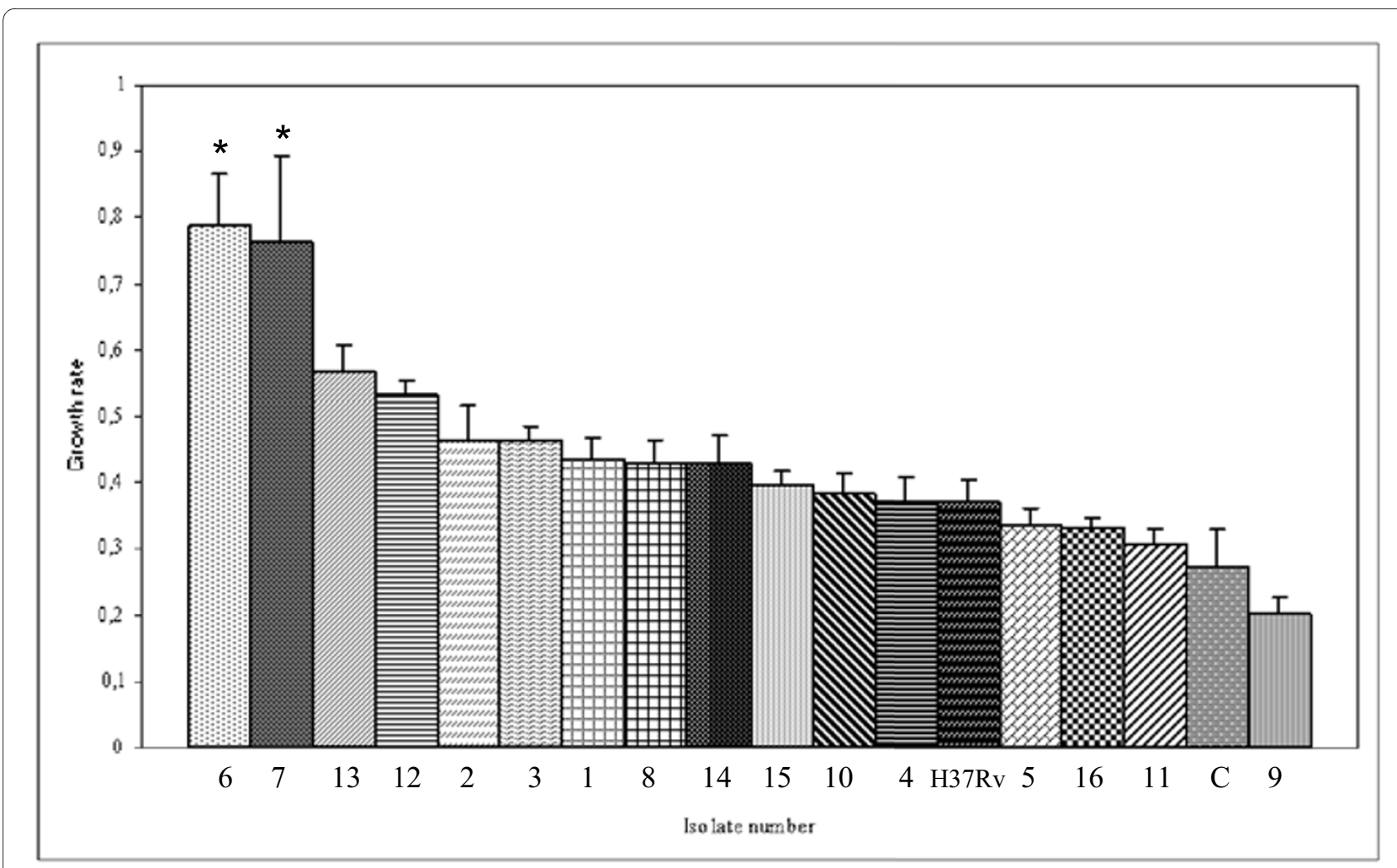

Figure 2 Intracellular growth rate in differentiated THP-1 cells. Cells were infected with 16 Beijing isolates, the control strain H37Rv, and a nonBeijing strain (C). Intracellular growth was expressed as the growth rate (Y Axis), ie, the slope of the function of $\log _{10}$ CFU values during the infection period. CFUs were determined 3 hours after infection and at days 1, 4, and 7. Results are expressed as the mean \pm standard error of the three independent experiments per strain. Asterisks indicate isolates with significantly higher intracellular growth rates $(P<0.05)$.

We attempted to characterize the infective features of the Beijing isolates in our sample by assaying a selection of isolates. We enriched the sample to be assayed in the infectivity model with additional Beijing isolates from another setting (Tuscany, Italy) in the Mediterranean area that had features, namely clustered strains, which were underrepresented in our area. As the Beijing lineage was the only genotype showing a steady expansion in Tuscany with frequent clustering (involving immigrants and autochthonous patients) [15], we included several isolates from this area in our sample.

To characterize the infective features of the Beijing isolates, an in vitro infection model using differentiated THP-1 cells was applied, which has been considered a good macrophage model [31-35] and which validity was proved after demonstrating that THP-1 cells differentiated with PMA express CD14, an antigen considered a marker for macrophages [36]. This model is also a good alternative for evaluation of the infectivity of MTB $[10,37,38]$.

Although the number of isolates in our study is small to draw general conclusions, an interesting finding was that the isolates showed heterogeneous infective behaviour, with a wide range of intracellular growth rates. Two iso- lates showed the highest growth rates and stood out significantly from the others.

We tested cytokine production in the in vitro infections, focusing on TNF- $\alpha$ and IL-10 as the main representatives of the Th- 1 and Th- 2 responses. In our model, the levels of cytokines always increased after infection, indicating that the assay, although activated by the addition of PMA, is not saturated. It allowed a measuring window to identify different infective behaviours among the strains analyzed. Indeed, it allowed us to efficiently measure the increases, in or maintenance or contention of cytokine production after infection caused by specific strains, which was our aim. We observed that infection with the isolates with the highest intracellular replication levels elicited the lowest TNF- $\alpha$ levels in THP-1-infected cells. At early stages of infection, these isolates induced significantly lower TNF- $\alpha$ production than the other isolates, and maintained this level until the end of infection, thus indicating failure to correctly induce the cytokine-dependent Th1-type protective immune response. Other authors have also observed a wide range of intracellular replication rates among Beijing isolates and an inverse association between intracellular replication levels and TNF- $\alpha$ production [30,39]. 
A

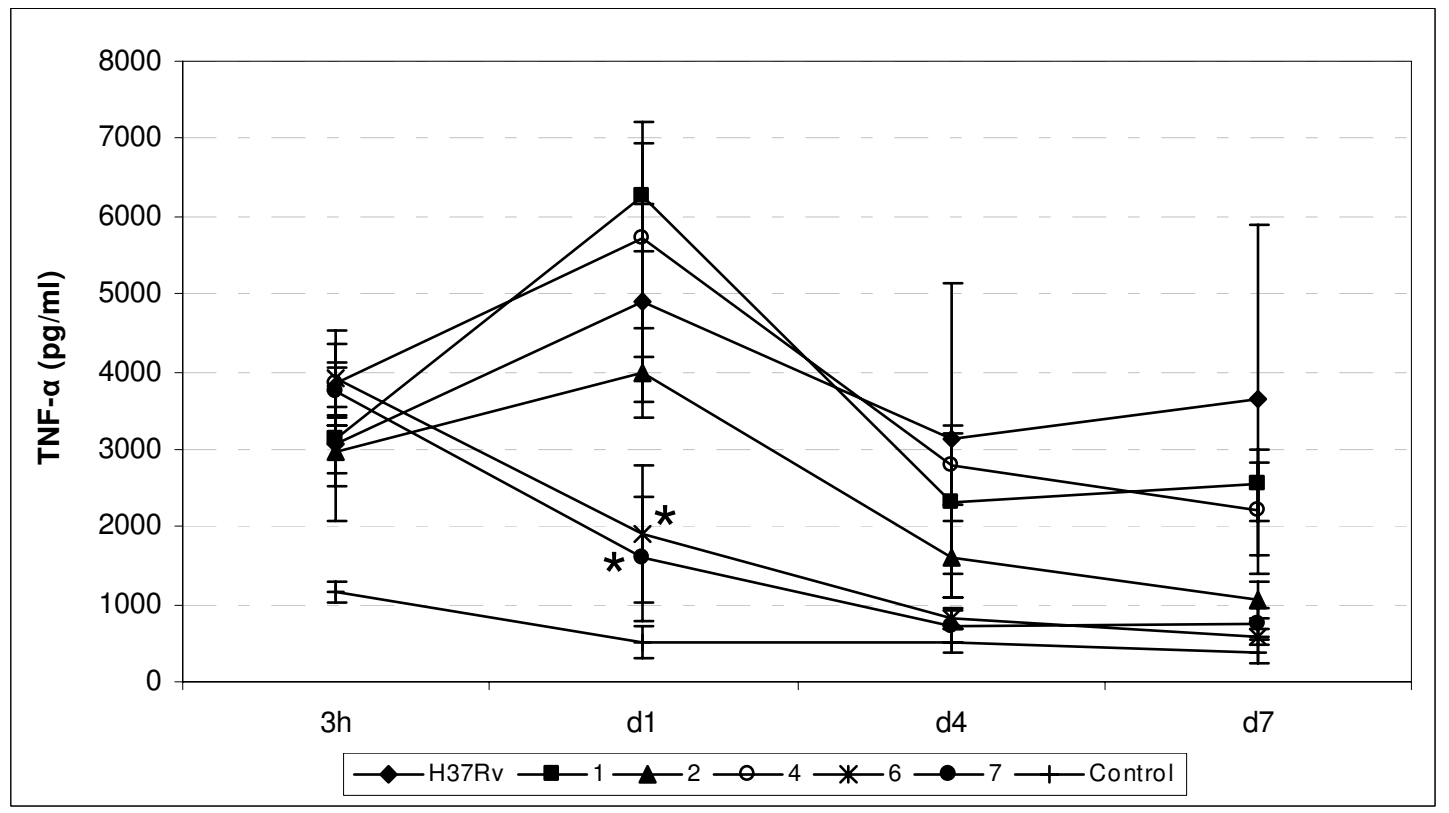

B

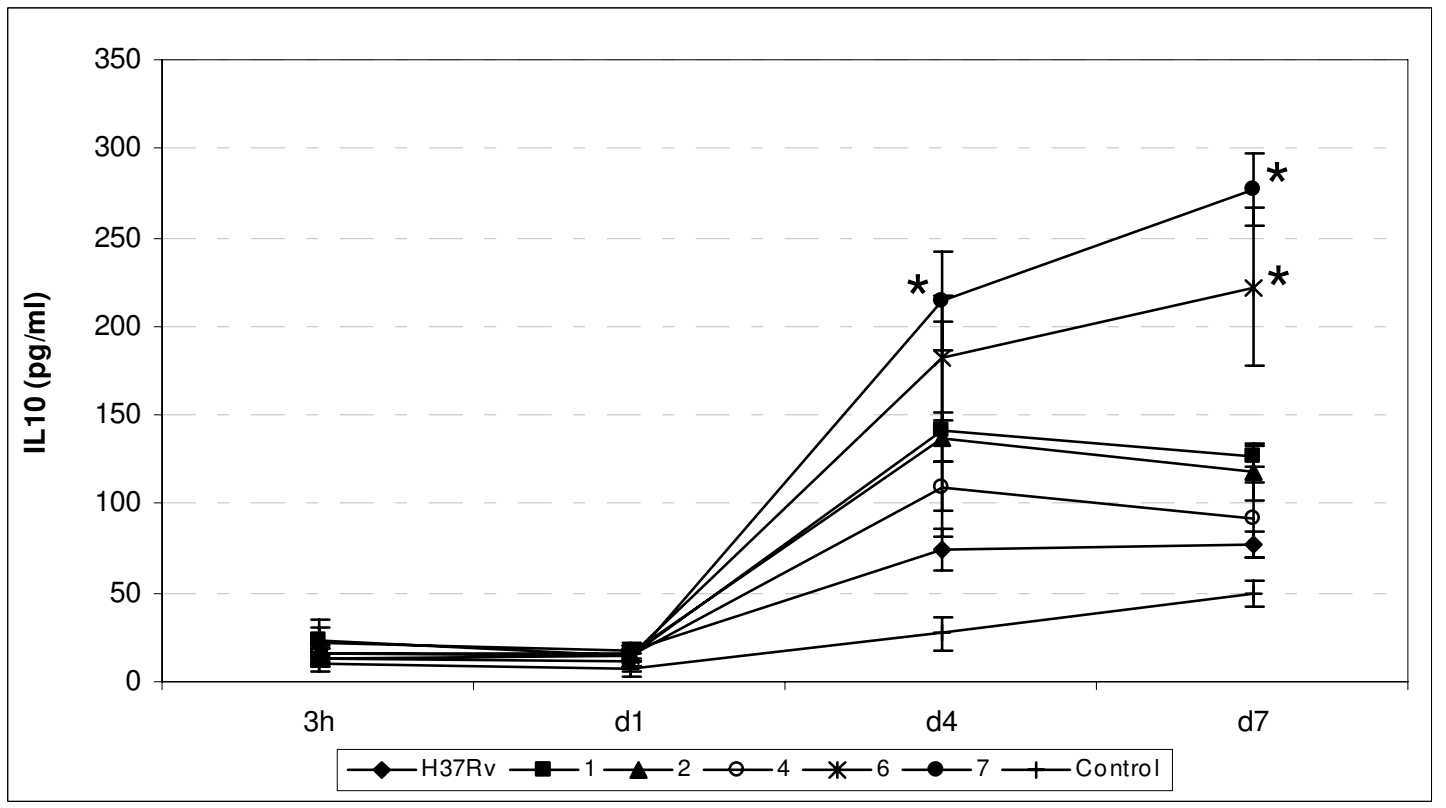

Figure 3 Cytokine production by differentiated THP-1 cells infected with strains representatives of different intracellular growth rates. Levels of TNF-a (panel A) and IL-10 (panel B) were determined in culture supernatants 3 hours after infection and at days 1, 4, and 7. Data are expressed as the mean \pm standard error of three independent experiments per strain. Asterisks indicate significantly different $(P<0.05)$ cytokine production. Control: Non-infected control cells.

Furthermore, low-virulence strains are associated with a more vigorous immune response with high levels of type 1 cytokines (TNF- $\alpha$, IFN- $\gamma$, IL-12) $[10,13,40]$. These data suggest that the infective advantage of Beijing strains should not be considered as an intrinsic feature of the lin- eage, but as a characteristic of certain representatives. These findings are highly relevant, as the outcome of the infection is related to the ability of MTB to regulate the induction of cytokines that are essential for the development of an efficient immune response [41]. As shown by 
our study and others, the virulent Beijing representatives induced high production of proinflammatory cytokines, which is quickly controlled, thus decreasing their levels and giving rise to a more effective infection. Phenol glycolipid (PGL), has recently been proposed as a virulence factor in Beijing strains [12]. This molecule can inhibit the release of key inflammatory effector molecules in vitro and has been considered responsible for the hypervirulent phenotype of Beijing strains, both in murine and rabbit infection models [12,42]. The different sub-groups of the Beijing lineage have recently been shown to contain different percentages of PGL-producing strains [18]; therefore, other factors could determine the hypervirulence of certain Beijing strains. As most of the isolates in our study belonged to sub-group 3 , it was not possible to explore in depth the relationships between infectivity and PGL production. However, isolates belonging to subgroup 3 displayed different intracellular growth rates. The only representative belonging to sub-group 4 (with the highest percentage of PGL-producing strains) showed the highest intracellular replication levels. Therefore, according to Reed et al [18], it would be very interesting to evaluate PGL production in these isolates to determine whether their hypervirulent phenotype (high intracellular replication rates, low production of TNF- $\alpha$ ) could correlate with the synthesis of this complex glycolipid.

Some studies have analyzed the relationship between intracellular growth and transmissibility [40,43], and concluded that the extensive spread of an MTB strain correlated with its high capacity to replicate, which is considered a marker of virulence. We studied intracellular replication levels of clustered and orphan strains, and observed no significant differences in growth rates or TNF- $\alpha$ production. In addition, the two isolates with the highest growth rates were orphan isolates. Therefore, virulence features are not always associated with clustered/ orphan status.

Clustered/orphan status could be a consequence of bacterial factors, although epidemiological features must also be taken into consideration. In this sense, it is interesting to mention our findings for the isolates corresponding to the strains involved in the Gran Canaria outbreak. The three patients infected with this strain had lived on Gran Canaria Island before arriving in Madrid, and there was a three-year interval between each diagnosis. This cluster seems more likely to be a coincidental finding in Madrid of three cases infected on Gran Canaria Island than a recent transmission in Madrid. Other than these cases, no secondary cases involving this genotype have been found since the last one (2006), which is the opposite of the explosive spread of the same strain on Gran Canaria Island. The lack of secondary cases by this genotype after its first detection in Madrid could suggest that epidemiological features more than bacteriological features (virulence, transmissibility, infectivity) could have been responsible for the Gran Canaria outbreak. Consistent with this explanation, the representative of this cluster did not show any replicative advantage or control over the immune response, and, therefore, this strain should not be considered especially virulent or transmissible.

\section{Conclusions}

In summary, we provide an outline of the genotypic and infective features of Beijing isolates identified in Spain and Italy. We show the low representativeness of this lineage in the study population, the association between the lineage and immigration, and the lack of association with resistant phenotypes. The infective profile of the Beijing isolates was markedly heterogeneous, suggesting the existence of certain highly virulent representatives in a nonhomogeneous lineage. In our sample, we did not find a correlation between virulence and phylogenetic group or resistance. A correlation between in vitro infectivity and the clustered/orphan status of the isolates was not found, which could reflect the complex process that infection/ transmission is with a potential role for patient-related factors (economical, social, epidemiological aspects). The Beijing strain which was extensively transmitted on Gran Canaria Island displayed a completely different epidemiological behaviour in Madrid, and did not show a highly infective phenotype in vitro. Various factors, both bacterial and epidemiological, seem to be behind the success and higher prevalence of Beijing strains compared with the other genetic lineages of MTB. The specific role that these factors could play in different contexts must be clarified before establishing general assumptions about the Beijing lineage.

\section{Methods \\ MTB strains}

Clinical samples were processed according to standard methods and grown in Lowenstein-Jensen slants (Becton Dickinson, Sparks, Maryland, USA). During the period 2002-2007, we received for genotyping a total of 2391 MTB cultures from two population-based studies in Spain between 2004 and 2008 [44,45]: 1872 isolates were from five urban areas in Madrid (6,081,689 inhabitants) and 519 were from Almeria (south-eastern Spain 646,633 inhabitants).

We also included, exclusively for the infectivity assays, eight Beijing isolates from a previous study performed from 2002 to 2004 in Tuscany (central Italy, 3,600,000 inhabitants) [15]. 


\section{Identification and genetic characterization of Beijing strains}

Spoligotyping was performed following the manufacturer's instructions (Isogen, Netherlands). The Beijing genotype was assigned on the basis of the spoligotype. In particular, isolates with spoligotype patterns characterized by deletion of spacers 1-34 were defined as "typical" Beijing, whereas isolates with additional deletion of one or more of the last nine spacers were defined Beijing-like according to the criteria of the international database SpolDB4 [22].

To confirm this identification of Beijing isolates by spoligotyping and also to refine the genetic characterization of the Beijing isolates, the $p k s 15 / 1$ gene and thegenomic deletions RD105, RD181, RD150, and RD142 were analyzed.

An intact $p k s 15 / 1$ gene has been reported to be a marker of the Beijing lineage [4,17], whereas a 7-bp deletion has been found for non-Beijing isolates. We purified DNA using standardized methods [46] to verify the marker by amplification and DNA sequencing [4] using an ABI-PRISM 310 sequencer (Lab Centraal B.V., Haarlem, NL).

The genomic deletions RD105, RD181, RD150, and RD142, which sub-classify the Beijing lineage, were identified by PCR using primers and conditions described elsewhere [5].

\section{Molecular typing methods}

DNA extraction and restriction fragment length polymorphism typing with the insertion sequence IS6110 (IS6110-RFLP) were performed according to standard methods [46]. Computer-assisted analysis of IS6110 fingerprints was carried out using Bionumerics 5.1 software (Applied Maths, Kortrijk, Belgium). Mycobacterial interspersed repetitive unit-variable number tandem repeat typing (MIRU-VNTR) was performed by amplifying the 15 MIRU-VNTR loci as described elsewhere [47], with some modifications [48]. The number of repetitions in each locus was calculated by applying the corresponding conversion tables (P. Supply, personal communication). The MIRU type was defined after combining the results for the 15 loci in the following order: MIRU4, MIRU26, MIRU40, MIRU10, MIRU16, MIRU31, Mtub04, ETRC, ETRA, Mtub30, Mtub39, QUB4156, QUB11b, Mtub21, and QUB26.

Five additional loci were added to the MIRU15 set: QUB11a and QUB18 [19,20], QUB3232 [19], and VNTR3820 and VNTR4120 [28,49], which were selected based on the high polymorphism found for the Beijing clade when applying these loci [28]. They were all amplified using simplex polymerase chain reaction (PCR) with primers described elsewhere $[19,28,50]$. Dimethyl sulfoxide (Sigma-Aldrich) was added in the amplification reac- tions for VNTR3820 and VNTR4120 (8\%) and QUB11a, QUB18, and QUB3232 (12\%). The sizes of the PCR products were calculated after electrophoresis in $2 \%$ agarose gels (MS8 agarose; Pronadisa, Madrid, Spain) for 17.5 hours at $45 \mathrm{~V}$ (for products under $800 \mathrm{bp}$ ) or 22 hours (for larger products). Assignation of alleles was based on table sizes kindly provided by Dr. Tomotada Iwamoto (Microbiology Dep., Kobe Health Institute, Japan) and on data published elsewhere $[19,20,28,49]$.

In certain cases, the large size for some products obtained at loci QUB11a, VNTR3820, and QUB3232 did not allow accurate assignation of alleles. In these cases, we only could estimate that the number of repetitions was higher than $20(>20)$. When we observed products differing in size in groups of isolates with more than 20 repetitions, we sub-labeled them $>20 \mathrm{a},>20 \mathrm{~b},>20 \mathrm{c}$ and $>20 d$.

For the analysis by MIRU-VNTR of the isolates sharing RFLP pattern with the strain involved in the Gran Canaria outbreak (analyzed in Hospital Miguel Servet, Zaragoza), only the 15-loci format was applied and not the expanded set of five additional loci, because these have not been validated for interlaboratory comparisons due to low interlaboratory reproducibility.

\section{Cluster analysis}

Genotypic patterns were analyzed using Bionumerics 4.6 (Applied Maths, Belgium). Dendrograms were generated using the unweighted pair group method with arithmetic averages (UPGMA) and the Dice coefficient or the categorical coefficient for IS6110-RFLP and MIRU-15 analysis, respectively. RFLP clusters and orphan status were defined for isolates sharing identical fingerprints after analyzing the patterns for the 2391 MTB isolates from the population-based sample. MIRU clusters were defined for isolates sharing identical patterns.

\section{Susceptibility test}

Susceptibility testing with isoniazid, rifampin, streptomycin, pyrazinamide, and ethambutol was performed using the mycobacterial growth indicator SIRE system (Becton Dickinson, Sparks, Maryland, USA).

\section{Cell cultures}

The human promonocytic cell line THP-1 was obtained from the American Type Culture Collection (TIB-202; Manassas, Virginia, USA). Cell cultures were maintained in modified RPMI 1640 + L-glutamine (Gibco, Grand Island, NY) supplemented with $10 \%$ fetal bovine serum (Gibco, Grand Island, NY), $10 \mathrm{mM}$ HEPES, and $50 \mu \mathrm{g} / \mathrm{ml}$ gentamicin (Gibco, Grand Island, NY). Cultures were maintained at 7-10 $\times 10^{5}$ cells $/ \mathrm{ml}$ and incubated at $37^{\circ} \mathrm{C}$ in $5 \% \mathrm{CO}_{2}$ in a humidified incubator. In order to ensure that we are working with a macrophage model, THP-1 
cells were differentiated to adherent macrophages by the addition of $200 \mathrm{nM}$ phorbol myristate acetate (PMA) (Sigma, St. Louis, MO) for 3 days at $37^{\circ} \mathrm{C}$ in $5 \% \mathrm{CO}_{2}$.

\section{Cell infection}

Cells were infected as described elsewhere [10], with slight modifications. Briefly, differentiated THP-1 cells seeded in 24-well flat-bottom tissue culture plates were washed and the medium was replaced to remove PMA and gentamicin 2 hours before the addition of bacteria. Cells were infected with a multiplicity of infection of 2-10 bacteria per cell and incubated for 3 hours at $37^{\circ} \mathrm{C}$ in $5 \%$ $\mathrm{CO}_{2}$. After incubation, monolayers were thoroughly washed with phosphate-buffered saline to remove extracellular bacteria and fresh medium was added. To evaluate the bacterial growth, supernatants were aspirated and monolayers were lysed with $0.5 \%$ Nonidet P40 (Roche Diagnostics, Mannheim, Germany) at 3 hours and days 1, 4 , and 7 after infection. Serial 10-fold dilutions of cellular lysates were plated on Middlebrook $7 \mathrm{H} 11$ plates and incubated for 3 weeks at $37^{\circ} \mathrm{C}$ in $5 \% \mathrm{CO}_{2}$, and colonies were counted. Intracellular growth was expressed as the growth rate, which is the slope of the function of $\log _{10}$ $\mathrm{CFU}$ values throughout the infection period (3 hours and days 1, 4, and 7). Three or more independent experiments were performed for each assayed strain.

\section{Cytokine analysis}

Culture supernatants from control and infected THP-1 cells were harvested after 3 hours and on days 1, 4, and 7, frozen at $-70^{\circ} \mathrm{C}$, and assayed using an enzyme-linked immunosorbent assay (ELISA) kit according to the manufacturer's instructions (BD Biosciences, Lincoln Park, NJ) to measure levels of tumor necrosis factor alpha (TNF- $\alpha$ ) and interleukin 10 (IL-10).

\section{Statistical analysis}

Three independent experiments were performed per strain. The means and standard errors were determined for each measurement in both intracellular growth and cytokine production. One-way analysis of variance with repetitive measures was used to determine $\mathrm{P}$ values, which were adjusted using the Bonferroni method. All the comparisons were carried out using the program SPSS 17.0.

\section{Competing interests}

The authors declare that they have no competing interests.

\section{Authors' contributions}

MA designed and performed all the experiments related to pks15/1, RDs and infectivity assays, analyzed the results, produced the first version of the MS and was involved in the correction of the MS. NA performed the molecular-epidemiology study, analyzed the results and collaborated in the production of the first version of the MS. CG provided a selection of MTB strains from Tuscany, Italy and critically reviewed the final version of the MS. MML and members from the INDAL-TB group, coordinated the molecular epidemiological study in
Almeria. MH performed the IS6110-RFLP and spoligotyping assays and analyzed the results. SS obtained and provided the IS6110-RFLP and MIRU-15 data for the Beijing isolates involved in the outbreak of G. Canaria and collaborated in the comparative analysis of these data with those obtained in Madrid. MJRS performed all the microbiological procedures. EB critically reviewed the final version of the MS. DGV designed the study, supervised all the experimental work, analyzed the results, corrected and produced the final version of the MS. All the authors read and approved the final version of the MS

\section{Acknowledgements}

This study was partially funded by the Fondo de Investigaciones Sanitarias (FIS060882; FIS061467; FIS06/90490; 06/90357), Junta de Andalucía (0453/06, 151/05), and the Instituto de Salud Carlos III (CIBER Enfermedades Respiratorias CB06/06/0058 and the Spanish Network for the Research in Infectious Diseases [REIPI RD06/0008]). N.A.R. received a grant from the Consejería de Educación de la Comunidad de Madrid and the European Social Fund (3334/2004). We are grateful to Joaquin Navarro from the Immunology Department in Gregorio Marañón Hospital for assessing us with the cytokine assays and to the INDALTB group in Almería for the recruitment of cases and compilation of clinical data. We are grateful to Thomas O'Boyle for editing and proofreading the final version of the manuscript.

\section{Author Details}

1Servicio de Microbiología Clínica y Enfermedades Infecciosas, Hospital Gregorio Marañón, Madrid, Spain, ${ }^{2}$ Unidad Central de Análisis Molecular (UCAM), Hospital Gregorio Marañón, Madrid, Spain, ${ }^{3}$ CIBER Enfermedades Respiratorias (CIBERES), Spain, 4Dipartimento de Patologia Sperimentale, Biotecnologie Mediche, Infettivologia ed Epidemiologia, Università de Pisa, Pisa, Italy, ${ }^{5}$ Complejo Hospitalario Torrecárdenas, Almería, Spain and ${ }^{6}$ Instituto Aragonés de Ciencias de la Salud, Zaragoza

Received: 24 December 2009 Accepted: 25 May 2010

Published: 25 May 2010

\section{References}

1. WHO: Global tuberculosis control: surveillance, planning, financing WHO report 2008. WHO/HTM/TB/2008.393.Geneva. 2008.

2. Frieden TR, Sterling TR, Munsiff SS, Watt CJ, Dye C: Tuberculosis. Lancet 2003, 362(9387):887-899.

3. Kremer K, Glynn JR, Lillebaek T, Niemann S, Kurepina NE, Kreiswirth BN, Bifani PJ, van Soolingen D: Definition of the Beijing/W lineage of Mycobacterium tuberculosis on the basis of genetic markers. J Clin Microbiol 2004, 42(9):4040-4049.

4. Constant P, Perez E, Malaga W, Laneelle MA, Saurel O, Daffe M, Guilhot C Role of the pks15/1 gene in the biosynthesis of phenolglycolipids in the Mycobacterium tuberculosis complex. Evidence that all strains synthesize glycosylated p-hydroxybenzoic methyl esters and that strains devoid of phenolglycolipids harbor a frameshift mutation in the pks15/1 gene. J Biol Chem 2002, 277(41):38148-38158.

5. Tsolaki AG, Gagneux S, Pym AS, Goguet de la Salmoniere YO, Kreiswirth BN, Van Soolingen D, Small PM: Genomic deletions classify the Beijing/ W strains as a distinct genetic lineage of Mycobacterium tuberculosis. J Clin Microbiol 2005, 43(7):3185-3191.

6. Bifani PJ, Mathema B, Kurepina NE, Kreiswirth BN: Global dissemination of the Mycobacterium tuberculosis W-Beijing family strains. Trends Microbiol 2002, 10(1):45-52.

7. Glynn JR, Whiteley J, Bifani PJ, Kremer K, van Soolingen D: Worldwide occurrence of Beijing/W strains of Mycobacterium tuberculosis: a systematic review. Emerg Infect Dis 2002, 8(8):843-849.

8. European Concerted Action on new generation genetic markers and techniques for the epidemiology and control of Tuberculosis. Beijing/ W genotype Mycobacterium tuberculosis and drug resistance. Emerg Infect Dis 2006, 12(5):736-743.

9. Samper S, Iglesias MJ, Rabanaque MJ, Gomez LI, Lafoz MC, Jimenez MS, Ortega A, Lezcano MA, Van Soolingen D, Martin C: Systematic molecular characterization of multidrug-resistant Mycobacterium tuberculosis complex isolates from Spain. J Clin Microbio/ 2005, 43(3):1220-1227.

10. Theus SA, Cave MD, Eisenach KD: Intracellular macrophage growth rates and cytokine profiles of Mycobacterium tuberculosis strains with different transmission dynamics. J Infect Dis 2005, 191(3):453-460. 
11. Lopez B, Aguilar D, Orozco H, Burger M, Espitia C, Ritacco V, Barrera L, Kremer K, Hernandez-Pando R, Huygen K, et al:: A marked difference in pathogenesis and immune response induced by different Mycobacterium tuberculosis genotypes. Clin Exp Immunol 2003, 133(1):30-37.

12. Reed MB, Domenech P, Manca C, Su H, Barczak AK, Kreiswirth BN, Kaplan G, Barry CE: A glycolipid of hypervirulent tuberculosis strains that inhibits the innate immune response. Nature 2004, 431(7004):84-87.

13. Manca C, Reed MB, Freeman S, Mathema B, Kreiswirth B, Barry CE, Kaplan G: Differential monocyte activation underlies strain-specific Mycobacterium tuberculosis pathogenesis. Infect Immun 2004, 72(9):5511-5514.

14. Caminero JA, Pena MJ, Campos-Herrero MI, Rodriguez JC, Garcia I, Cabrera P, Lafoz C, Samper S, Takiff H, Afonso O, et al.: Epidemiological evidence of the spread of a Mycobacterium tuberculosis strain of the Beijing genotype on Gran Canaria Island. Am J Respir Crit Care Med 2001 164(7):1165-1170

15. Lari N, Rindi L, Bonanni D, Rastogi N, Sola C, Tortoli E, Garzelli C: Three-year longitudinal study of genotypes of Mycobacterium tuberculosis isolates in Tuscany, Italy. J Clin Microbiol 2007, 45(6):1851-1857.

16. Koksalan OK, Kilicaslan Z, Zanlier G, Guzel R, Seber E: Prevalence of Beijing genotype Mycobacterium tuberculosis strains in Istanbul. Int J Tuberc Lung Dis 2006, 10(4):469-472.

17. Chaiprasert A, Yorsangsukkamol J, Prammananan T, Palittapongarnpim $P$, Leechawengwong M, Dhiraputra C: Intact pks15/1 in non-W-Beijing Mycobacterium tuberculosis isolates. Emerg Infect Dis 2006, 12(5):772-774

18. Reed MB, Gagneux S, Deriemer K, Small PM, Barry CE: The W-Beijing lineage of Mycobacterium tuberculosis overproduces triglycerides and has the DosR dormancy regulon constitutively upregulated. J Bacteriol 2007, 189(7):2583-2589.

19. Le Fleche P, Fabre M, Denoeud F, Koeck JL, Vergnaud G: High resolution, on-line identification of strains from the Mycobacterium tuberculosis complex based on tandem repeat typing. BMC Microbiol 2002, 2:37.

20. Wada T, Maeda S, Hase A, Kobayashi K: Evaluation of variable numbers of tandem repeat as molecular epidemiological markers of Mycobacterium tuberculosis in Japan. J Med Microbio/ 2007, 56(Pt 8):1052-1057.

21. Direccion general de Salud Publica. 2007. Registro regional de casos de tuberculosis de la Comunidad de Madrid. Informe del año 2006. Boletin epidemiologico de la Comunidad de Madrid 13(12):4-41.

22. Brudey K, Driscoll JR, Rigouts L, Prodinger WM, Gori A, Al-Hajoj SA, Allix C, Aristimuno L, Arora J, Baumanis V, et al:: Mycobacterium tuberculosis complex genetic diversity: mining the fourth international spoligotyping database (SpolDB4) for classification, population genetics and epidemiology. BMC Microbiol 2006, 6:23

23. Garcia de, Viedma D, Chaves F, Inigo J: New route of importation of Mycobacterium tuberculosis Beijing genotype. Emerg Infect Dis 2006, 12(1):169-170.

24. Codina G, Vidal R, Martin-Casabona N, Miravitlles M, Martin C: Multidrug resistant tuberculosis caused by 'W'-related strains in three immunocompetent foreign-born patients. Int J Tuberc Lung Dis 1999 3(1):82-84.

25. WHO: Anti-tuberculosis drug resistance in the world. Fourth global report. WHO/HTM/TB/2008.394. Geneva. 2008.

26. Kremer K, van-der-Werf MJ, Au BK, Anh DD, Kam KM, van-Doorn HR, Borgdorff MW, van-Soolingen D: Vaccine-induced immunity circumvented by typical Mycobacterium tuberculosis Beijing strains. Emerg Infect Dis 2009, 15(2):335-339.

27. Kremer K, van Soolingen D, Frothingham R, Haas WH, Hermans PW, Martin C, Palittapongarnpim P, Plikaytis BB, Riley LW, Yakrus MA, et al.: Comparison of methods based on different molecular epidemiological markers for typing of Mycobacterium tuberculosis complex strains: interlaboratory study of discriminatory power and reproducibility. $J$ Clin Microbiol 1999, 37(8):2607-2618.

28. Iwamoto T, Yoshida S, Suzuki K, Tomita M, Fujiyama R, Tanaka N, Kawakami Y, Ito M: Hypervariable loci that enhance the discriminatory ability of newly proposed 15-loci and 24-loci variable-number tandem repeat typing method on Mycobacterium tuberculosis strains predominated by the Beijing family. FEMS Microbiol Lett 2007, 270(1):67-74.

29. Kremer K, Au BK, Yip PC, Skuce R, Supply P, Kam KM, van Soolingen D: Use of variable-number tandem-repeat typing to differentiate
Mycobacterium tuberculosis Beijing family isolates from Hong Kong and comparison with IS6110 restriction fragment length polymorphism typing and spoligotyping. J Clin Microbio/ 2005, 43(1):314-320.

30. Theus S, Eisenach K, Fomukong N, Silver RF, Cave MD: Beijing family Mycobacterium tuberculosis strains differ in their intracellular growth in THP-1 macrophages. Int J Tuberc Lung Dis 2007, 11(10):1087-1093.

31. Puig-Kroger A, Serrano-Gomez D, Caparros E, Dominguez-Soto A, Relloso M, Colmenares M, Martinez-Munoz L, Longo N, Sanchez-Sanchez N, Rincon M, et al:: Regulated expression of the pathogen receptor dendritic cell-specific intercellular adhesion molecule 3 (ICAM-3)grabbing nonintegrin in THP-1 human leukemic cells, monocytes, and macrophages. J Biol Chem 2004, 279(24):25680-25688.

32. Tsuchiya S, Kobayashi Y, Goto Y, Okumura H, Nakae S, Konno T, Tada K: Induction of maturation in cultured human monocytic leukemia cells by a phorbol diester. Cancer Res 1982, 42(4):1530-1536.

33. Li Y, Mohammad RM, al-Katib A, Varterasian ML, Chen B: Bryostatin 1 (bryo1)-induced monocytic differentiation in THP-1 human leukemia cells is associated with enhanced c-fyn tyrosine kinase and M-CSF receptors. Leuk Res 1997, 21(5):391-397.

34. Vey E, Zhang JH, Dayer JM: IFN-gamma and 1,25(OH)2D3 induce on THP-1 cells distinct patterns of cell surface antigen expression, cytokine production, and responsiveness to contact with activated T cells. J Immunol 1992, 149(6):2040-2046.

35. Bombara C, Ignotz RA: TGF-beta inhibits proliferation of and promotes differentiation of human promonocytic leukemia cells. J Cell Physiol 1992, 153(1):30-37.

36. Zhou J, Zhu P, Jiang JL, Zhang Q, Wu ZB, Yao XY, Tang H, Lu N, Yang Y, Chen ZN: Involvement of CD147 in overexpression of MMP-2 and MMP9 and enhancement of invasive potential of PMA-differentiated THP-1. BMC Cell Biol 2005, 6(1):25.

37. Theus SA, Cave MD, Eisenach KD: Activated THP-1 cells: an attractive model for the assessment of intracellular growth rates of Mycobacterium tuberculosis isolates. Infect Immun 2004, 72(2):1169-1173.

38. Stokes RW, Doxsee D: The receptor-mediated uptake, survival, replication, and drug sensitivity of Mycobacterium tuberculosis within the macrophage-like cell line THP-1: a comparison with human monocyte-derived macrophages. Cell Immunol 1999, 197(1):1-9.

39. Wong KC, Leong WM, Law HK, Ip KF, Lam JT, Yuen KY, Ho PL, Tse WS, Weng $\mathrm{XH}$, Zhang WH, et al:: Molecular characterization of clinical isolates of Mycobacterium tuberculosis and their association with phenotypic virulence in human macrophages. Clin Vaccine Immuno/ 2007, 14(10):1279-1284.

40. Theus SA, Cave MD, Eisenach K, Walrath J, Lee H, Mackay W, Whalen C, Silver RF: Differences in the growth of paired Ugandan isolates of Mycobacterium tuberculosis within human mononuclear phagocytes correlate with epidemiological evidence of strain virulence. Infect Immun 2006, 74(12):6865-6876.

41. Chacon-Salinas R, Serafin-Lopez J, Ramos-Payan R, Mendez-Aragon P, Hernandez-Pando R, Van Soolingen D, Flores-Romo L, Estrada-Parra S, Estrada-Garcia I: Differential pattern of cytokine expression by macrophages infected in vitro with different Mycobacterium tuberculosis genotypes. Clin Exp Immunol 2005, 140(3):443-449.

42. Tsenova L, Ellison E, Harbacheuski R, Moreira AL, Kurepina N, Reed MB, Mathema B, Barry CE, Kaplan G: Virulence of selected Mycobacterium tuberculosis clinical isolates in the rabbit model of meningitis is dependent on phenolic glycolipid produced by the bacilli. J Infect Dis 2005, 192(1):98-106

43. Zhang M, Gong J, Yang Z, Samten B, Cave MD, Barnes PF: Enhanced capacity of a widespread strain of Mycobacterium tuberculosis to grow in human macrophages. J Infect Dis 1999, 179(5):1213-1217.

44. Alonso Rodriguez N, Chaves F, Inigo J, Bouza E, Garcia de Viedma D, Andres S, Cias R, Daza R, Domingo D, Esteban J, et al:: Transmission permeability of tuberculosis involving immigrants, revealed by a multicentre analysis of clusters. Clin Microbiol Infect 2009, 15(5):435-442.

45. Alonso-Rodriguez N, Martinez-Lirola M, Sanchez ML, Herranz M, Penafiel T, Bonillo Mdel C, Gonzalez-Rivera M, Martinez J, Cabezas T, Diez-Garcia LF, et al:: Prospective universal application of mycobacterial interspersed repetitive-unit-variable-number tandem-repeat genotyping to characterize Mycobacterium tuberculosis isolates for fast identification of clustered and orphan cases. J Clin Microbiol 2009, 47(7):2026-2032. 
46. van Embden JD, Cave MD, Crawford JT, Dale JW, Eisenach KD, Gicquel B, Hermans P, Martin C, McAdam R, Shinnick TM, et al: Strain identification of Mycobacterium tuberculosis by DNA fingerprinting: recommendations for a standardized methodology. J Clin Microbiol 1993, 31(2):406-409

47. Supply P, Allix C, Lesjean S, Cardoso-Oelemann M, Rusch-Gerdes S, Willery $E$, Savine $E$, de Haas $P$, van Deutekom $H$, Roring $S$, et al.: Proposal for standardization of optimized mycobacterial interspersed repetitive unit-variable-number tandem repeat typing of Mycobacterium tuberculosis. J Clin Microbiol 2006, 44(12):4498-4510.

48. Alonso-Rodriguez N, Martinez-Lirola M, Herranz M, Sanchez-Benitez M, Barroso P, Bouza E, Garcia de Viedma D: Evaluation of the new advanced 15-loci MIRU-VNTR genotyping tool in Mycobacterium tuberculosis molecular epidemiology studies. BMC Microbiol 2008, 8:34.

49. Smittipat N, Billamas P, Palittapongarnpim M, Thong-On A, Temu MM Thanakijcharoen $P$, Karnkawinpong $O$, Palittapongarnpim $P$ : Polymorphism of variable-number tandem repeats at multiple loci in Mycobacterium tuberculosis. J Clin Microbiol 2005, 43(10):5034-5043.

50. van Deutekom H, Supply P, de Haas PE, Willery E, Hoijng SP, Locht C, Coutinho RA, van Soolingen D: Molecular typing of Mycobacterium tuberculosis by mycobacterial interspersed repetitive unit-variablenumber tandem repeat analysis, a more accurate method for identifying epidemiological links between patients with tuberculosis. J Clin Microbiol 2005, 43(9):4473-447.

doi: $10.1186 / 1471-2180-10-151$

Cite this article as: Alonso et al., Characterization of Mycobacterium tuberculosis Beijing isolates from the Mediterranean area BMC Microbiology 2010 10:151

Submit your next manuscript to BioMed Central and take full advantage of:

- Convenient online submission

- Thorough peer review

- No space constraints or color figure charges

- Immediate publication on acceptance

- Inclusion in PubMed, CAS, Scopus and Google Scholar

- Research which is freely available for redistribution

Submit your manuscript at www.biomedcentral.com/submit
Ciomed Central 